\title{
Gefahr droht CAP-Patienten vor allem durch resistente gramnegative Erreger
}

\author{
Wie sieht die derzeitige Resistenzlage bei Atemwegskeimen aus? Es gibt keinen Grund zur Panik, \\ Probleme bereiten vor allem resistente gramnegative Erreger.
}

\begin{abstract}
Multiresistente Keime als Ursache ambulant erworbener Pneumonien (CAP) sind in Deutschland nach wie vor selten. Zur größten Bedrohung könnte in Zukunft die Zunahme resistenter gramnegativer Bakterien werden, vor allem von ESBL-Bildnern, sagte Prof. Tobias Welte aus Hannover.

Bei grampositiven Erregern von akuten Atemwegsinfektionen sei die Situation in Deutschland viel günstiger als in vielen südeuropäischen Ländern, so Welte. Dies liege vermutlich vor allem an dem weit häufigeren Antibiotikaeinsatz in Südeuropa in den vergangenen Jahrzehnten sowohl bei Menschen als auch in der Tierzucht. Die Konsequenz aus der weltweit sehr unterschiedlichen Resistenzlage sollten nationale Empfehlungen zur Therapie bei Atemwegsinfektionen sein, die sich an der lokalen Resistenzlage orientieren, betonte der Infektiologe. Therapieempfehlungen aus den USA könnten nicht auf Europa übertragen werden und bewährte Rezepte in Nordeuropa könnten in Südeuropa ungeeignet sein.
\end{abstract}

\section{Welches Antibiotikum für welches Bakterium?}

Wie sieht die aktuelle Situation bei Atemwegskeimen aus? Keine Probleme würden in den meisten europäischen Ländern nach wie vor Streptokokken machen, so Welte, mit einem Anteil von über $50 \%$ seien sie die häufigsten Erreger von CAP. Die Penicillinresistenz ist in Europa mit Ausnahme von Spanien gering. Gäbe es Resistenzen, könnten sie durch Betalaktam-Antibiotika überwunden werden. Der Anteil Makrolid-resistenter Streptokokken beträgt in Nordeuropa bis zu 20\%, in einigen Ländern Südeuropas aber schon bis zu 50\%. Für die Prognose der Patienten haben diese Resistenzen aber praktisch keine Bedeutung, sagte Welte.

Viel relevanter sind hingegen Makrolid-resistente Mykoplasmen, die auf die Prognose von CAP-Patienten einen signifikanten Einfluss hätten. Während der Anteil von Makrolidresistenzen bei Mycoplasma pneumonia in Deutschland noch unter 5\% liege, seien in Südostasien bereits bis zu 90\% der Keime resistent. Dies läge nicht nur am hohen Antibiotikaverbrauch in Asien, sondern auch an weit verbreitete Generika schlechter Qualität mit zu geringem Wirkstoffanteil, so Welte.

Bei Staphylococcus aureus haben Methicillin-Resistenzen mit rund 20\% in den meisten europäischen Ländern ein Plateau erreicht. In einigen Ländern wie Großbritannien und Frankreich gingen die Resistenzen in den letzten Jahren sogar zurück. Bei Methicillin-empfindlichen Erregern sollte kein Vancomycin eingesetzt werden, betonte Welte. Die Prognose der so behandelten Patienten sei schlechter als bei Einsatz eines BetalaktamAntibiotikums. Bei Methicillin-resistenten Staphylococcus aureus (MRSA) hänge die Wahl der Therapie vom Ort der Infektion ab. Vancomycin sei gut bei Patienten mit Bakteriämie, aber nicht gut geeignet bei Pneumonie-Patienten. Bei Linezolid sei es umgekehrt. Eine dramatische Zunahme gebe es von Vancomycin-resistenten Enterococcus faecium, so Welte weiter, aber dies sei für die Lunge ein untergeordnetes Problem.

\section{Doppeltes Komplikationsrisiko bei ESBL-Infektion}

Als größte Bedrohung bei CAP-Patienten nannte er gramnegative Erreger, etwa Extended-Spectrum-Betalaktamase, kurz ESBL-bildende Bakterien, deren Resistenzen im vergangenen Jahrzehnt stark zugenommen hätten. Die Wahrscheinlichkeit von Komplikationen verdoppelt sich bei Patienten mit ESBL-Infektion. Bei Patienten mit Carbapenem-Resistenz - in Griechenland und Italien liegt der Anteil bereits bei 50\% - sei mit einer vierfach erhöhten Mortalität zu rechnen. Auch PseudomonasInfektionen der Lunge korrelierten mit einer deutlich verschlechterten Prognose, allerdings sei die Resistenzsituation nicht so schlecht. Jedoch werden wegen zum Teil hoher Resistenzen gegen Chinolone wie Ciprofloxacin diese Substanzen nicht mehr zur Monotherapie empfohlen.

Das Fazit von Welte: Es gibt bei der Resistenzlage von Atemwegskeimen derzeit keinen Grund zur Panik. Gefährdet durch resistente Erreger seien vor allem Patienten mit Risikofaktoren wie Komorbiditäten und chronischer Kolonisation. Roland Fath

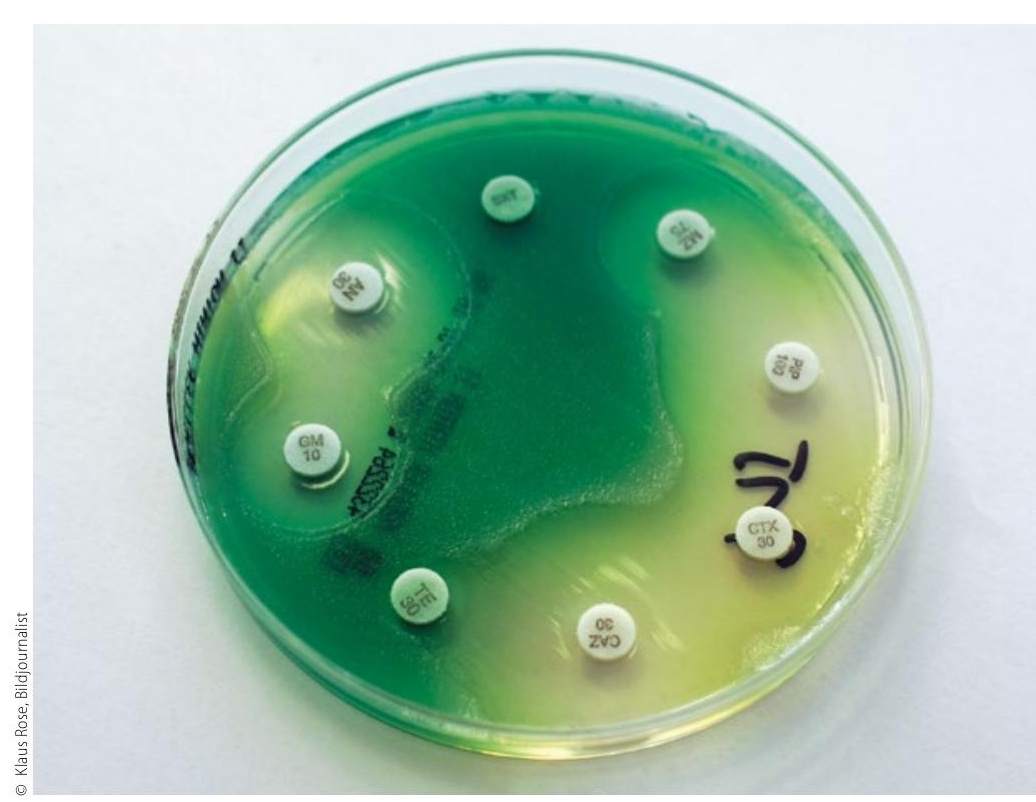

Insbesonders resistente gramnegative Atemwegskeime wie Pseudomonas aeruginosa können in der Klinik problematisch werden. 